\title{
A feasibility study of short message service text messaging as a surveillance tool for alcohol consumption and vehicle for interventions in university students
}

Simon C Moore ${ }^{1 *}$, Katherine Crompton ${ }^{2}$, Stephanie van Goozen ${ }^{3}$, Marianne van den Bree ${ }^{4}$, Julia Bunney ${ }^{5}$ and Emma Lydall ${ }^{1}$

\begin{abstract}
Background: Practitioners who come into contact with the intoxicated, such as those in unscheduled care, often have limited resources to provide structured interventions. There is therefore a need for cost-effective alcohol interventions requiring minimal input. This study assesses the barriers, acceptability and validity of text messaging to collect daily alcohol consumption data and explores the feasibility of a text-delivered intervention in an exploratory randomised controlled trial.
\end{abstract}

Methods: Study I. Participants $(n=82)$ completed the initial online screening survey and those eligible were asked each day, for 157 days via text message, to reply with the number of alcohol units consumed the previous day. Analyses compared standard measures of hazardous consumption with self-report alcohol use. Attrition and sampling biases were examined. Study I included secondary exploratory analyses using data from 70 participants to determine associations between events (including Christmas and other celebratory occasions) and consumption. Study I further included the thematic analysis of semi-structured interview data and assessed the feasibility of and barriers to surveillance and interventions delivered through text messaging. Developing findings from Study I, Study II developed an exploratory randomised control trial that delivered a single message on monthly alcohol expenditure in order to assess effect size and test generalisability.

Results: Self-report alcohol consumption data was significantly associated with FAST and AUDIT scores. Attrition from the study was not associated with greater alcohol use. Greater alcohol use was observed on Fridays, Saturdays and Wednesdays as were notable celebratory events. Interview data indicated that text messaging was acceptable to participants and preferred over email and web-based methods. The exploratory randomised controlled trial suggested that a simple text delivered intervention might be effective in eliciting a reduction in alcohol consumption in a future trial.

Conclusions: The ubiquity of mobile telephones and the acceptability of text messaging suggests that this approach can be developed as a surveillance tool to collect high frequency consumption data to identify periods of vulnerability and that it can offer a platform through which targeted interventions can be delivered.

Keywords: Short message service (SMS), Alcohol, Students, University, Intervention

\footnotetext{
* Correspondence: mooresc2@cardiff.ac.uk

${ }^{1}$ Violence \& Society Research Group, School of Dentistry, Cardiff University,

CF14 4XY Cardiff, Wales

Full list of author information is available at the end of the article
} 


\section{Background}

This study assesses the feasibility of using mobile telephone text messages to both survey and moderate alcohol use. Text messaging is an ubiquitous mode of communication and short text messages (SMS) are found in a range of communication platforms. The World Bank estimates the number of mobile cellular subscriptions per 100 people (including post- and pre-paid subscriptions) in 2010 was 90.15 in the USA, 130.34 in the UK, and, globally, 78.16, a figure that increased from 15.54 globally in 2001 [1]. Text messages are limited to 140 bytes or 160 English alphabet characters. In North America, the market analysts Nielsen estimate 13-17 year olds made on average 231 telephone calls compared to sending and receiving 3,952 (for females) and 2,815 (for men) messages each month [2]. This ubiquity of mobile technology and therefore access to SMS brings opportunities for practitioners to exploit this platform and so develop protocols for surveillance and intervention $[3,4]$. SMS offers a particularly attractive vehicle as the cost of mobile equipment is modest compared to, for example, personal computers and there is some evidence to suggest that the more vulnerable and those of a low socio-economic status are more likely to have access to and use mobile telephones compared to personal computers [5,6]. Furthermore, brief text messages that are pushed from the server to the recipient are increasingly the default mode of communication across a range of platforms, including Twitter and Facebook. An understanding of the benefits of using short text messages in public health will therefore potentially generalise across a number of platforms and devices.

Mobile telephones are portable, usually accompany users and provide a constant opportunity to communicate and intervene at times when recipients are most vulnerable to misuse alcohol [5]. For example, identifying when the risk of alcohol misuse is greatest (there is a strong association between sporting and other celebratory events and the incidence of alcohol-related harm [7]) means targeted messages could be delivered at those times. While telephony has provided a platform from which the use of technology in healthcare has developed, with further rapid development expected in the near future $[8,9]$, SMS has, to date, received only limited attention $[3,4]$. The bulk of research has focused on internet delivered projects, which require access to personal computers [10-14]. While there is some interest in developing bespoke applications for smart phones, such as a piece of software that runs on a smartphone and is tailored to a specific health-related intervention, these applications do not typically push data to the recipient and instead require that they actively engage, reducing the immediacy of any message. Therefore, SMS provides an immediate, popular means of communication that could provide opportunities to both identify when drinkers are at greatest risk and deliver interventions at these times.

Evidence indicates that the SMS platform can deliver successful interventions in healthcare generally and to address alcohol-related harms specifically. Studies of SMS use have focused on treatment non-compliance, the dissemination of test results and tackling clinic nonattendance through providing appointment reminders [15]. Fjeldsoe, Marshall and Miller [3] reviewed studies published between 1990 and 2008 that evaluated healthrelated interventions delivered using SMS. They identified 14 studies in total that targeted tobacco use $(n=4)$ and clinical care $(n=10)$, the latter included physical activity, obesity, diabetes, asthma, hypertension and bulimia. SMS has also been used with more difficult to reach groups, such as patients with severe mental illness where real-time data was collected in order to facilitate case management [5]. SMS has also been effective in settings with limited resources. For example, SMS has been used to collect data from caregivers on their adherence to anti-retroviral therapy guidelines in Uganda [16]. A cluster randomised controlled trial in Kenya used SMS to improve caseworkers' adherence to malaria case-management practice [17], yielding strong short-term and sustained improvements. While there is evidence indicating SMS provides an information delivery platform that can improve health outcomes there may also be opportunities to collect alcohol consumption data to both inform intervention content (e.g., determine the characteristics of individuals' drinking patterns to guide subsequent interventions) or to collect information useful in determining intervention success.

Kuntsche and Robert [18] demonstrated that SMS could be used to collect alcohol consumption data from a young adult (predominantly student) sample in Switzerland. They collected data over four weekends and obtained a 75\% participant retention rate. However, it is known that students often participate in heavy mid-week drinking $[19,20]$ and it is also likely that student drinking varies across the academic year according to course commitments and celebratory events. It would therefore be useful to determine whether SMS can be used to collect high frequency (daily) alcohol consumption surveillance data over a longer time period than that used by Kuntsche and Robert [18] and to assess whether this platform can identify times of greatest risk to inform targeted interventions.

The SMS platform has also been used to survey and intervene with young adults identified in Emergency Departments and who have a history hazardous alcohol use. Suffoletto [21] recruited young adults in an Emergency Department for a small-scale feasibility study of a randomised controlled trial. The authors report good compliance rates with $95 \%$ of those involved responding to weekly consumption questions. However, this is in marked contrast to the use of Personal Digital Assistants, handheld 
computers that are in addition to what study participants might normally carry. Serre [22] studied the temporal associations between a range of substances and alcohol. Those misusing marijuana yielded a compliance rate of $31 \%$ in response to repeated questions delivered by Personal Digital Assistants. Electronic diaries have also been used successfully to explore the relationship between alcohol misuse and hangovers [23] as well as more general negative consequences of alcohol use [24].

The current paper focussed on undergraduate students. Students in the UK are among the heaviest drinkers in the country but their alcohol consumption patterns may not yet be engrained, providing opportunities for early intervention. Using mixed methods we sought to examine factors relating to recruitment and attrition from a SMS survey of drinking habits, and to test the validity of high frequency alcohol consumption data collected through SMS. Comparisons were made with data collected through traditional on-line surveys (which were also used to collect additional socio-economic information from participants) the completion of which was also prompted through SMS.

In summary, the first (Study I) objective was to (a) quantitatively assess the validity of self-report alcohol consumption data collected through a SMS text messaging survey against established measures of hazardous alcohol use and to assess whether the characteristics of respondents' alcohol use predicted attrition from the SMS survey. Secondary analyses of the survey data were conducted in order to (b) determine whether SMS messaging could identify periods when participants were most vulnerable to misuse alcohol. Study I also (c) qualitatively assessed the acceptability of SMS text messaging, both as a survey of alcohol use and as a vehicle for intervention. Study II built on Study I and explored the feasibility of delivering a feedback intervention using estimated monthly alcohol expenditure. The goal was to test whether a randomised controlled trial was feasible and to provide early insights into the nature and impact of a brief intervention delivered through SMS.

The overall aim of the studies reported here was to inform a Phase II trial of a text delivered alcohol intervention. The research therefore follows existing guidance on how such formative research should be developed and evaluated [25] in which there is a strong emphasis on testing intervention feasibility, identifying barriers to implementation, developing theory and providing realistic sample size estimates [25]. Mixed methods were used to assess intervention delivery, acceptability to participants, define outcome measures, and provide prospective sample size estimates. Study I is formative, developing the platform required in an exploratory randomised controlled trial (Study II) that both provides sample size estimates for a phase two trial and tests generalisability to a non-student sample.

\section{Methods}

All aspects of the research reported here were reviewed and approved by the Dental Research Ethics Committee, Cardiff University.

\section{Study I: Validation, feasibility and acceptability of collecting high frequency consumption data Participants}

Participants were recruited at a University "Freshers' Fayre", an event in the first week of the academic year at which local businesses, University societies and other organisations seek to recruit first year students ("freshers"). A booth was set up at this event and flyers were distributed that provided information about the study. Recruitment materials included written informed consent and a brief questionnaire. All those attending the Freshers' Fayre were eligible to complete this form, irrespective of their subsequent eligibility for inclusion in the study (alcohol consumers who were over 18 years of age), thus providing information that could be used to assess any selection biases relating to alcohol use in later analyses. Potential participants were incentivised by an opportunity to win a laptop, or cash equivalent in shopping vouchers. Participants in the secondary analysis of qualitative data were also drawn from those consenting to participate. Figure 1 shows the flow of participants through the study.

Participants in the qualitative study were those who had completed initial registration materials. An invitation to participate was sent by SMS asking whether they would be interested in assisting in return for payment (£10). For the qualitative arm, sampling continued to saturation but the sample was stratified by gender and level of SMS study involvement: opt in and opt out (if they did or did not respond to at least one SMS survey question), and male and female. Five males and four females were recruited who responded to SMS messages at least once and then actively opted out (denoted "opt out" in the Results section) and four males and five females who participated continuously (denoted "opt in" in the Results section).

\section{Materials}

\section{Initial recruitment}

A brief two-part form was used at recruitment that included an information sheet for participants to keep and a second sheet that collected respondents' mobile telephone number, year of study, the Fast Alcohol Screening Test (FAST) [26], a question on whether they drank alcohol ("yes, regularly", "yes, occasionally", "never, but might in the future" and "never have and never will") and written consent. The information sheet further contained instructions on how participants could leave the study and stop receiving further SMS messages (by replying to any study SMS message with "STOP"). Also included were instructions on how to respond to daily text messages 


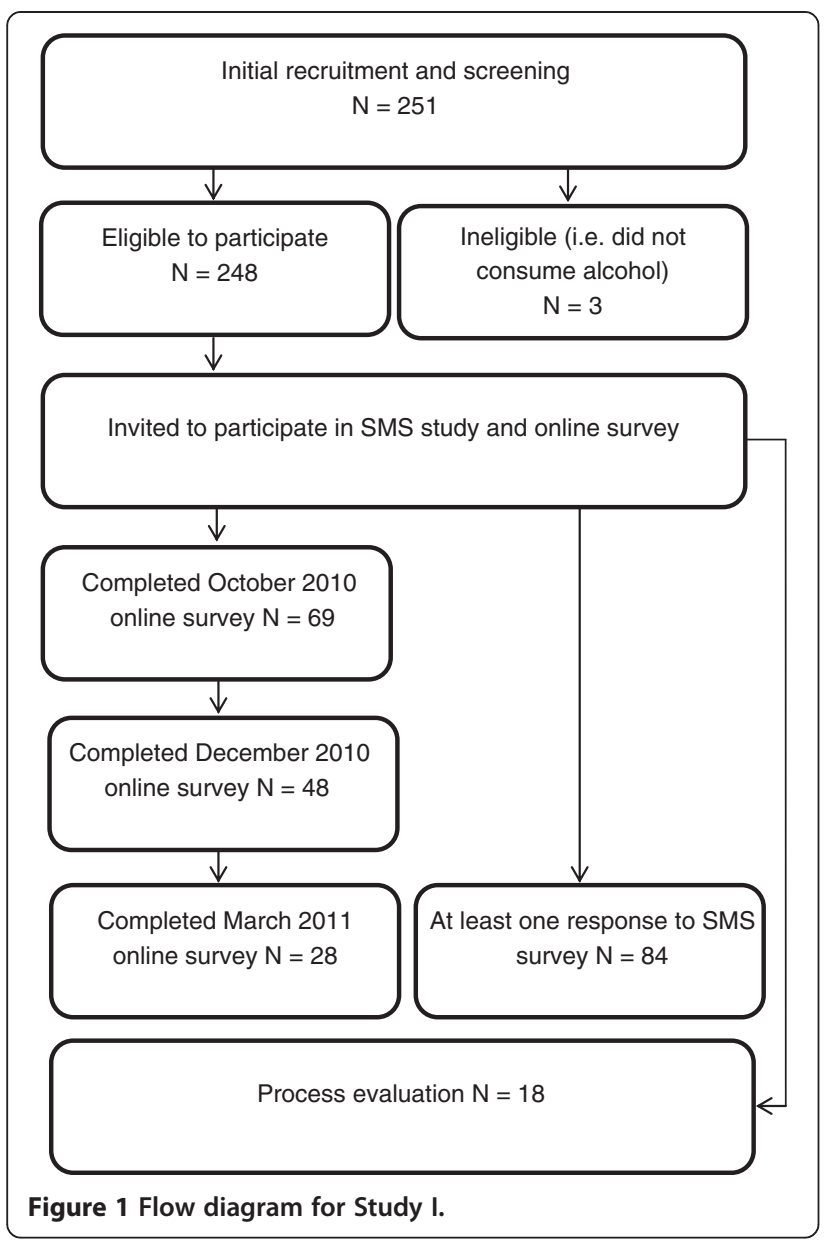

(by replying with the number of standard UK units consumed the previous day) and a definition of a unit of alcohol was provided. The study website address was provided. This website repeated instruction on how participants could leave the study, the definition of an unit of alcohol, researcher contact information, links to the online surveys (see below) and information on study incentives.

An online survey was created that collected further information from participants. Questions included their mobile phone number (used also as an identification field so that multiple data sources could be matched), FAST [26], the Alcohol Use Disorders Identification Test (AUDIT; [27]), of which FAST is a subset, age and gender, year and course of study, where they were living (e.g. at home with their parents, in private rented accommodation, etc.), tobacco use, marital status, whether they had dependent children, and total monthly income. The first online survey included questions relating to social perceptions and delay discounting, these data are not reported here.

Two measures of hazardous alcohol use are used in the current study, the FAST [26] and the AUDIT [27]. Audit is a ten question test that determines whether a respondent's alcohol use is hazardous or not. Because AUDIT takes time to complete and is therefore not always suitable in clinical practice (one place where SMS delivered interventions might be developed), FAST was developed as a shorter alternative, using four questions from the AUDIT. Both AUDIT and FAST are typically used to triage patients according to need for alcohol intervention. Those drinkers at moderate risk are eligible and best suited for a brief intervention [28] whereas those at greater risk are best diverted to specialised alcohol health workers for more intensive treatment [29]. Both AUDIT and FAST are therefore used to allocate alcohol intervention resources efficiently, so that effectiveness and cost-efficiency are maximised. Given the current goal of developing SMS delivered interventions it is therefore appropriate that we characterise SMS derived consumption data using these standard tests.

A SMS text marketing organisation was contracted to to bulk deliver SMS messages at designated times. Questions were sent from a dedicated SMS number to which participants could respond for 22 weeks. The question "How many units of alcohol did you drink yesterday and last night? 1 unit = half pint beer, small wine, sngl shot [study URL] to quit, prizes, info" was sent by SMS every day. On a bi-monthly schedule a further message was sent with the internet address for an online survey. These additional surveys replicated the first online survey (see above) and allowed us to explore changes in indices such as the FAST.

For the qualitative study, 18 face to face interviews were conducted in public locations convenient to participants. Participants were paid $£ 10$ towards their travel expenses. All interviews were digitally recorded, anonymised and transcribed for analysis. All participants provided written informed consent. Interview themes included the acceptability and effectiveness of text messaging as a means of data collection and the feasibility of using text messaging as a means of delivering interventions.

\section{Procedure}

At the Freshers' Fayre respondents read the Participant Information Sheet, signed the consent form and completed the brief questionnaire. Eligible participants were sent an SMS message requiring them to access the first online survey on 18 October 2010. From 25 October 2010, 157 daily text messages on consecutive days were sent requesting consumption data for the preceding day. Initially, daily text messages were sent at 7 am but due to complaints that this was waking participants, this time was changed to $9 \mathrm{am}$ at the end of the study's fourth week (week 46 in Figure 2). In addition, and referring to Figure 2., technical issues meant that a subset of participants received messages twice while the remaining participants did not always receive messages at the scheduled time. This was corrected 


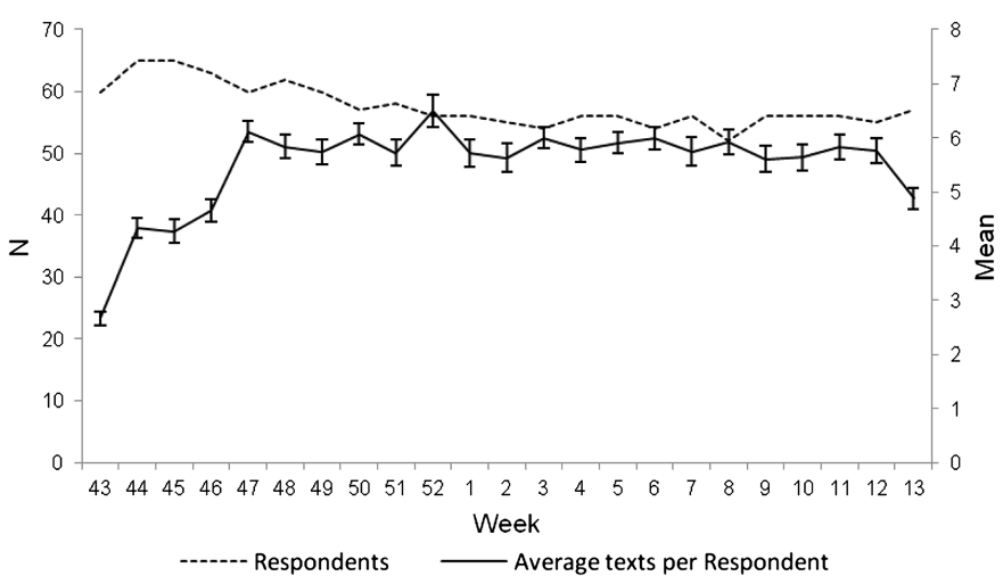

Figure 2 Average number of SMS responses per week (right side axis) and total number of respondents responding by week (left side axis). Text messages were sent to participants part way through week 43 and ended part way through week 13 . Week 1 is the first week in January.

at the end of the fourth week. Participants were informed that they could claim reimbursement for the text messages they sent at the end of the study. No participants requested reimbursement, probably due to the prevalence of mobile phone contracts with inclusive messaging. At initial recruitment participants were given the opportunity to enter into a prize draw for a laptop computer. Further incentives were a prize draw for $£ 200$ if they responded to bi-monthly invitations to complete the subsequent online surveys. No further attempts to enhance compliance were made. The SMS consumption messages were sent daily until 31 March 2011. Participants were sent the web address of the online survey through SMS, at the beginning of the study and this was repeated a further two occasions, equally spaced across the study period.

Semi-structured interviews were conducted with participants, both those who completed the SMS study and those who dropped out, to investigate their views on the use of this medium. The quantitative study was conducted first and preliminary analyses went forward to inform the themes explored in the qualitative study.

\section{Analysis}

The design of this study was longitudinal within-subjects. All analyses were conducted using Stata 12MP.

\section{Attrition and sampling biases}

Logistic regression assessed whether the FAST score collected at recruitment predicted completion of the first online survey. A t-test assessed differences in FAST score collected at recruitment for those that responded to SMS surveillance questions and those who did not. Spearman's rank correlation statistic explored associations between the number of SMS surveillance messages responded to and initial FAST score. Fixed effects (FE) Poisson regression was used to assess the relationship between units consumed and significant social events (including variation across the week).

\section{Additional exploratory analyses}

Pearson product moment correlation statistics were used to explore associations between FAST and AUDIT on the first online survey. Multiple linear regression was used to consider the relationship between FAST and average number of units consumed each day, controlling for gender. Multiple linear regression was also used to consider the relationship between FAST and average number of days alcohol was consumed, controlling for gender. Interpolation methods were used to construct time varying indices of hazardous alcohol use over the study period from both the FAST and the AUDIT. FE time series OLS models were used to assess the relationship between these indices and both daily units of alcohol consumed and a time series logit was used to assess the relationship between these indices and likelihood of drinking on a given day. FE logits were used to assess whether the likelihood of drinking on a given day was related to the preceding days level of alcohol consumption.

For the qualitative component, data were analysed using an inductive, thematic analysis, allowing themes to develop naturally, facilitating in depth exploration of each theme using descriptive and interpretive analysis [30]. Traditional Text Analysis was used to code and categorise developing themes [31].

Results from Study I informed the design and content of Study II. 
Study 2: A feasibility randomised control trial using SMS text messaging to provide feedback on alcohol expenditure

The purpose of this Study was to test the feasibility of a RCT designed to assess the impact of a single text message containing information on past alcohol expenditure on future consumption.

\section{Participants}

Participants were recruited by emailing students and nonstudents who had participated in previous university research studies and had expressed an interest in future research. Potential participants were incentivised by advertising an opportunity to be entered into a prize draw for one of four $£ 50$ high street vouchers. Participants in Study II had not participated in Study I. Figure 3 describes the flow of participants through the study.

\section{Materials}

The initial recruitment email contained brief information about the study and advertised the opportunity of being

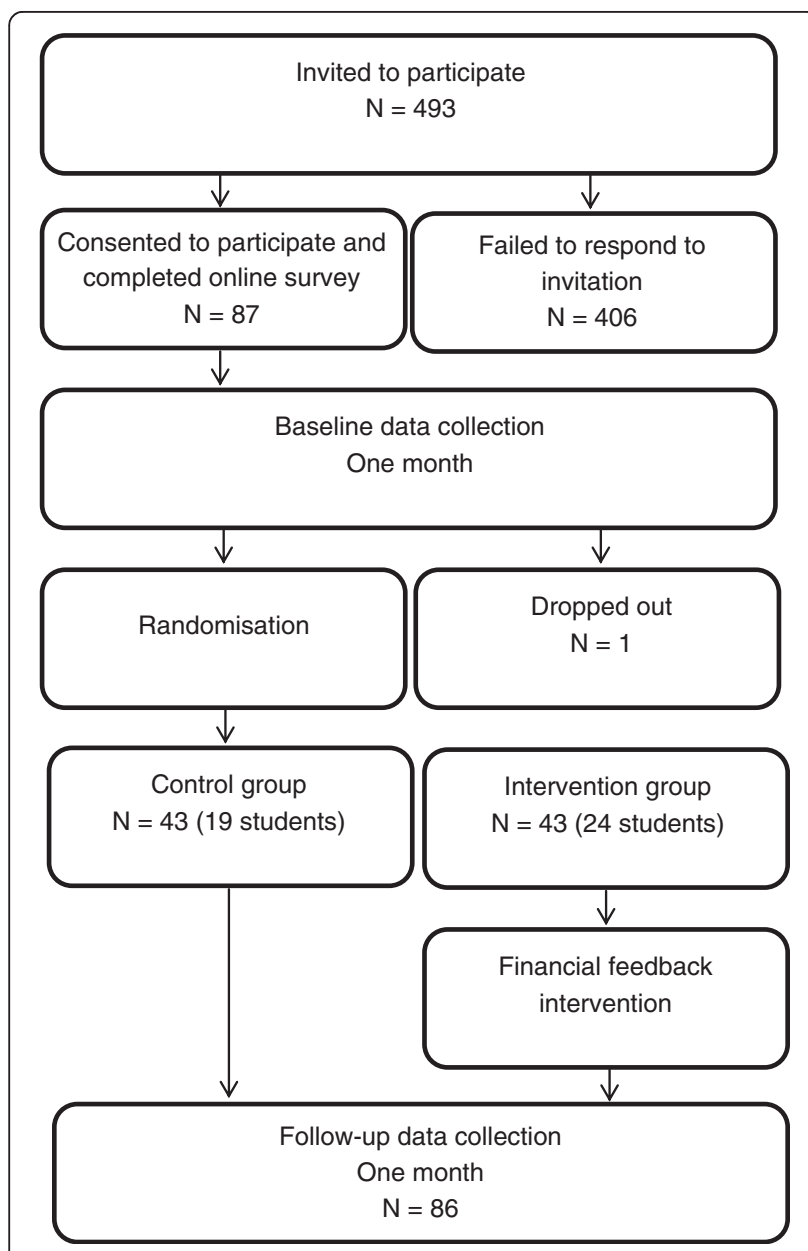

Figure 3 Consort diagram for the feasibility trial. entered into a free prize draw. It also contained a web address for a participant information sheet. This information sheet contained further information about the study, and clearly explained that participants would receive daily text messages for two calendar months, a development informed by the previous study in which some participants were not aware that they would receive daily text messages. It further explained how to respond to these messages (by replying with the number of standard units consumed on the previous day), and a definition of units. Also included were researcher contact details and instructions on how participants could leave the study and stop receiving further SMS messages (by replying 'STOP' to any study SMS message).

An online survey was created to collect information from people who decided to take part. Questions were identical to those used in the Study I online survey but for Study II social perceptions and delay discounting questions were not included. An additional question on occupational status was included, replacing the more detailed questions on student course of study and year of study. Participants were also asked questions about their usual drink, how much they normally spent on their usual drink both at home and in pubs/bars, and what proportion of their drinks were consumed at home. This was asked to enable a weighted estimation of their average expenditure per unit of alcohol.

The SMS text marketing organisation used for study I was contracted to deliver SMS messages at 11 am each day. Questions were sent from a dedicated number to which participants could respond. The question "How many units of alcohol did you drink yesterday and last night? 1 unit = half pint beer, small wine, sngl shot [study URL] to quit, prizes, info" was sent by SMS daily. The study website contained detailed instruction on how participants could leave the study and further information on the prizes they could win.

\section{Procedure}

A message was uploaded to the Cardiff University electronic Notice Board system, and an email was sent to further potential participants, advertising the study. Potential participants were able to access the Participant Information Sheet via an internet link. If they then wished to take part in the study they then completed the online survey.

Respondents were randomly assigned to an experimental group or a control group, stratified by gender and student status. From 15th May 2012 until 15th July 2012, all participants received daily text messages requesting consumption data for the preceding day. On 14th June 2012 (midway through data collection), participants in the experimental group were sent an estimation of their alcohol expenditure during the previous month. This was calculated using their reported alcohol consumption and their 
average unit price. The intervention message, 'Alcohol study: We estimate that you have spent $£ x$ on alcohol in the last month', was delivered via SMS. The short message containing expenditure on alcohol was the intervention.

\section{Analysis}

Analysis of variance was used to determine the relationship, if any, between treatment condition and alcohol consumption. Power analysis was then used to consider what sample size would be required to raise any effect to significance for a formal trial.

\section{Results}

\section{Study I: Validation and feasibility of collecting high frequency consumption data Descriptive statistics}

251 participants were recruited at the Fayre yielding an average FAST score of 3.96 ( $\mathrm{SD}=3.46)$. Three participants stated that they never have and never would drink alcohol; they were not invited to participate in the SMS study. Of the remaining 248 participants, 82 (51\% male, 92\% undergraduates, mean age 20.95 years, $\mathrm{SD}=2.90)$ completed the online survey in response to the initial text (sent October 2010 by SMS).

\section{Attrition}

Three participants completed the initial web survey twice, the second set of responses for these participants were dropped. A logistic regression analysis was used to assess the relationship between FAST score at recruitment and subsequent completion of the initial online survey, no relationship was observed $(\mathrm{OR}=1.03,95 \% \mathrm{CI}=0.96,1.12)$; 69 participants completed the FAST at recruitment, as well as the first online FAST and AUDIT. Pearson product moment correlation statistics indicate that all scores were strongly associated ( $r>0.85, p<0.0001$ for all comparisons). Participants were asked to complete three online surveys, once at the beginning and again in December 2010 and March 2011 at the end of the project; 82 participants completed and provided FAST and AUDIT scores in the first online survey, 48 in the second, and 28 completed the third online survey; 84 participants responded at least once to the daily SMS messages (one participant continued to respond beyond 31 March 2011, these data were discarded, and two participants responded to SMS messages despite not completing the first online survey). On average $86.25(\min =1, \max =156)$ responses were received from participants replying at least once to SMS text questions. A t-test on initial FAST score collected at recruitment for those who did not respond ( $\mathrm{n}=175$, mean FAST $=3.697, \mathrm{SD}=3.338)$ to SMS messages against those who responded at least once $(n=84$, mean FAST $=4.579$, $\mathrm{SD}=3.682)$ indicated responders had higher FAST scores $(\mathrm{t}=1.86, p<0.05)$. Furthermore, Spearman's rank measure of association suggested no relationship between FAST scores collected at recruitment and number of responses to SMS messages for those responding at least once or more $(\rho=-0.100, p=0.391)$. These results suggest that attrition is pronounced for online surveys prompted by SMS, whereas SMS responses remain stable over time (see Figure 2).

\section{Validation}

We computed the average number of units consumed and the proportion of days participants consumed alcohol. A multiple regression model indicated that a higher baseline FAST score ( $\mathrm{B}=0.392, p<0.01,95 \% \mathrm{CI} 0.180$ 0.604) was associated with a higher average number of units consumed whereas being female $(B=-1.823, p<0.05,95 \%$ CI $-3.360-0.293$ ) was associated with lower average number of units consumed. A second model indicated that a higher FAST score $(B=0.008, p=0.302,95 \%$ CI -0.0080 .025 ) was not statistically associated with the average number of days in which alcohol was consumed whereas being female $(B=-0.118, p<0.05,95 \%$ CI $-0.235-0.001)$ was associated with lower number of days drinking. These results provide external validity; indicating that the average quantity of alcohol consumed on each drinking occasion, recorded through SMS, is predicted by initial FAST scores, although not the frequency of drinking occasions.

Twenty eight participants completed all three online surveys and provided FAST and AUDIT scores as well as responded to SMS survey messages (min responses $=100$ ). To enable further modelling, a cubic spline interpolation algorithm was used to create smoothed FAST and AUDIT scores across the response period for these 28 participants. As there were fewer FAST and AUDIT readings taken over the course of the study, compared to daily SMS messages, we preferred to maximise the use of the SMS data to increase power. As this algorithm can, in some instances, yield interpolated values less than zero, values less than zero were replaced with zero (44 interpolated AUDIT scores and 30 interpolated FAST scores, out of a total of 3,365 data points for each, were replaced). Neither interpolated FAST scores $(B=0.025, p=0.834,95 \%$ CI $-0.2100 .260)$ nor interpolated AUDIT scores $(\mathrm{B}=0.157$, $p=0.059,95 \%$ CI -0.0060 .320 ) were significantly associated with self-report alcohol units consumed in FE linear models. A drinking frequency variable ( 0 if no alcohol was consumed, 1 if alcohol was consumed for each day) was derived. In FE logits interpolated AUDIT scores were positively associated with drinking likelihood $(B=0.100$, $p<0.01,95 \%$ CI 0.0350 .164 ) although interpolated FAST scores were not $(\mathrm{B}=0.063, p=0.175,95 \% \mathrm{CI}-0.0280 .155)$.

Figure 4 shows average alcohol consumption by day of week. The highest consumption occurred on Fridays (mean $=4.08$ units, SD =7.29), closely followed by Saturdays 


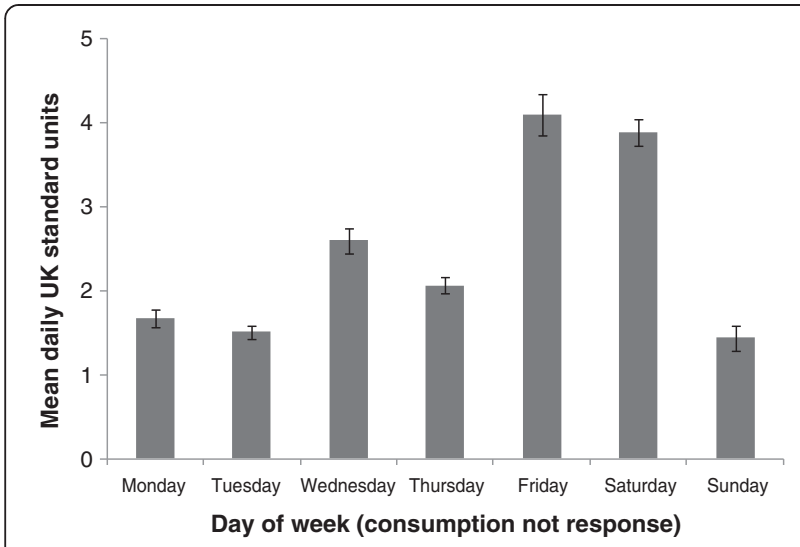

Figure 4 Mean self-reported units consumed by day of week with standard error bars of the mean.

(mean $=3.86$ units, SD=7.10). Of weekdays (SundayThursday inclusive), Wednesdays saw the heaviest alcohol consumption (mean $=2.57, \mathrm{SD}=5.63$ ), followed by Thursdays $($ mean $=2.08$ units, $\mathrm{SD}=5.55)$. Sundays saw the lowest alcohol consumption (mean $=1.44$ units, $\mathrm{SD}=3.87$ ).

Fixed-effects Poisson regression was used to determine the significance of this observed weekly variation. Binary (dummy) variables were assigned to each day of the week and Saturday was used as a reference category. Compared to Saturday, significantly fewer units were consumed on all other days of the week (Sunday: $B=-1.01,95 \%$ $\mathrm{CI}=-1.07-0.95$, Monday: $B=-0.81,95 \% \mathrm{CI}=-0.87-0.76$, Tuesday: $B=-0.91,95 \% C I=-0.97-0.85$, Wednesday: $B=-0.41,95 \% C I=-0.46-0.36$, Thursday: $B=-0.63$, $95 \% \mathrm{CI}=-0.68-0.58)$ except Fridays, which did not significantly differ from Saturday $(B=0.02,95 \% C I=-0.02$ $0.07)$. Sundays saw the lowest levels of consumption $(B=-1.01,95 \% C I=-1.07-0.95)$. The exponentiated coefficient on Sunday yields a rate ratio of 0.44 (95\% $\mathrm{CI}=0.340 .39)$ suggesting that on Sundays participants drank less than half the amount they drank on Saturdays.

Figure 5 shows daily alcohol consumption across the study period. Alcohol consumption was highest on New Year's Eve (mean $=12.51$ units, SD = 9.14) followed by the Welsh Varsity sporting event (mean $=7.36$ units, $\mathrm{SD}=10.70$ ). Alcohol consumption was also elevated on Christmas Eve (mean $=6.40$ units, $\mathrm{SD}=7.71)$, Christmas Day (mean $=6.18$ units, $S D=5.92$ ) and Boxing Day (mean $=6.70$ units, SD =9.30). The trend line in Figure 5 suggests a peak in alcohol consumption during late December and a trough in early January. Alcohol consumption during the 'twelve days of Christmas' (defined here as 21st December to 1st January) was greater (mean $=4.07$ units, $\mathrm{SD}=3.18$ ) compared to the rest of the study period (mean $=2.32$ units, $\mathrm{SD}=1.49$ ).
Longitudinal fixed-effects Poisson regression analysis confirmed this observation. A dummy variable described whether, for each day of the study, reported alcohol consumption fell within the "twelve days" $(=1)$ or outside $(=0)$ and further day of week dummies were included to control for the variation in consumption across a typical week. Significantly more alcohol was consumed during the 'twelve days of Christmas' compared to the rest of the study, irrespective of the days of the week that fell within the two time periods $(\mathrm{B}=0.53,95 \% \mathrm{CI}=0.48$ $0.57, \mathrm{p}<0.001)$. The corresponding rate ratio of 1.69 (95\% CI $=1.621 .77)$ suggests that participants drink $70 \%$ more during the holiday period.

A logit was used to consider whether the reported units of consumption on the preceding day influenced the likelihood of alcohol use. A derived binary variable described whether reported units consumed was zero (0) or greater than zero (1) and a FE logit indicated that the more units consumed the preceding day the less likely participants were to consume alcohol $(\mathrm{B}=-0.016, p<0.01$, $95 \%$ CI $-0.027-0.005)$, controlling for day of week effects. Using the time that SMS messages were sent and responses were received we calculated the delay between when the SMS message was sent and participants' response. A FE linear model indicated that a the more alcohol consumed the longer the response delay on the following day $(\mathrm{B}=0.019, p<0.001,95 \%$ CI 0.0100 .028$)$.

\section{Celebratory occasions}

The eight celebratory events were all associated with an increase in alcohol consumption. The highest mean units of alcohol were consumed on New Year's Eve, Welsh Varsity, Boxing Day, Christmas Eve, Christmas Day, Halloween, St Patrick's Day and Valentine's Day (Figure 6).

Each event was described by a dummy variable and day of week dummies were included as above. FE Poisson regression indicated that alcohol consumption was statistically greater during these events compared to other days of the week. The largest increase in drinking was seen on Boxing Day followed by Halloween, New Year's Eve, Welsh Varsity and Christmas Eve. The rate ratio of Boxing Day suggests respondents drank 6.57 (95\% CI $=5.777 .47, \mathrm{p}<.001)$ times what they would usually drink and on Halloween drank 4.18 (95\% CI = $3.594 .86, \mathrm{p}<.001)$ times what they would usually drink. Similarly, on New Year's Eve participants drank $3.88(95 \% \mathrm{CI}=3.534 .27, \mathrm{p}<.001)$ times their usual level of consumption and during Welsh Varsity students drank 3.51 (95\% CI = 3.12 3.96, p <.001) times usual consumption. Valentine's Day and St Patrick's Day had the smallest increases, 1.42 (95\% CI $=1.17$ $1.73, \mathrm{p}<.001)$ and $1.42(95 \% \mathrm{CI}=1.181 .69, \mathrm{p}<.001)$ respectively. 


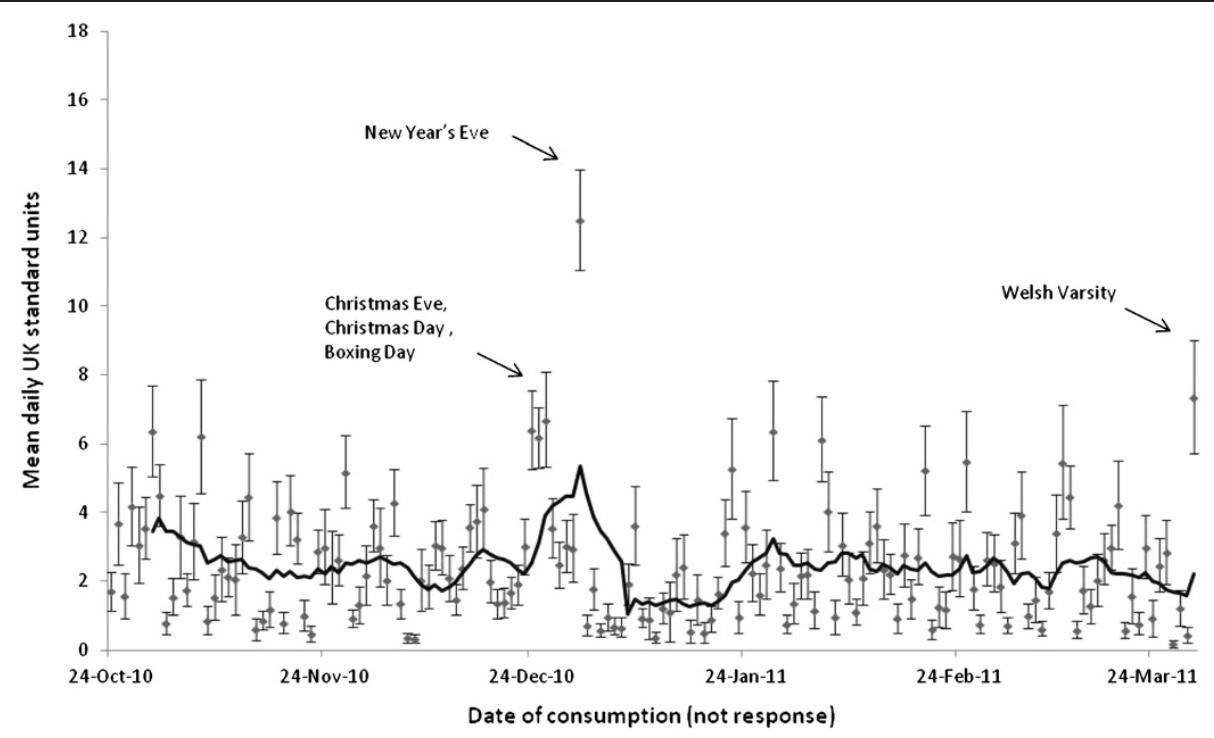

Figure 5 Mean self-reported units consumed daily across study period with standard error bars of the mean and a twelve point moving average (solid line).

\section{Qualitative analysis}

Results are organised according to the principal emergent themes. Interviews lasted between 17 and 55 minutes (average 32 minutes).

\section{SMS messaging}

With respect to attitudes towards SMS messaging in general, all participants regarded information through this medium positively and preferred SMS messages to telephone calls and electronic mail.

"It's really good. I think I like texts because sometimes, you know, you don't check your emails every day or, you know, log on to a computer, bit of an effort. [...] You get a text, you read a text" (male, opt in, VN550029).

"Well, [SMS is] certainly the easiest way. Emails don't always get checked and phone calls are almost too intrusive" (male, opt out, VN550039).

The perceived privacy afforded by SMS messages was explicitly referenced in one case.

"It was confidential, and like, you could guarantee it was confidential" (male, opt out, VN550038).

\section{SMS messaging frequency}

Twelve participants stated daily text messages were acceptable: "[the frequency] didn't really bother me" (male, opt

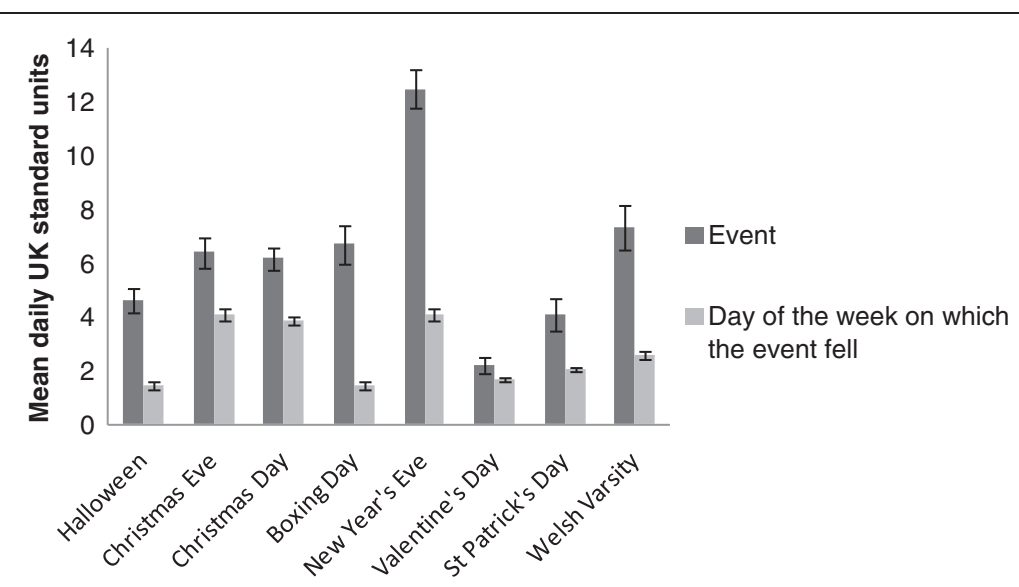

Figure 6 Mean self-reported units consumed on the 'twelve days of Christmas' and eight specific celebratory events (dark grey), compared with the mean number of units consumed on the day of week on which the event fell with standard error bars of the mean. 
out, VN550045), "daily was fine" (female, opt in, VN550033) and "the frequency was fine" (female, opt out, VN550040). Where participants objected to message frequency this was attributable to misaligned study expectations.

"I don't think I knew enough when I signed up that it was going to be so, erm, persistent" (female, opt out, VN550041).

\section{Message timing}

Message timing was one of the most significant barriers to participation. Messages were originally sent at $7 \mathrm{am}$, this was changed to 9 am after the fourth week of the study (end of week 46 in Figure 1), following complaints that the text messages were waking participants up.

"It was a bit off putting, a bit irritating, especially early in the morning. If it was in the day time it would have been fine, but it was always around $7 \mathrm{am}$. No, I don't think I wanted texting at 7 am in the morning, especially to be asked about my drinking habits... so the frequency of the texts themselves wasn't too bad... it was the timing of the texts rather than the number" (male, opt out, VN550039).

\section{Measures}

As with numerous studies, this study elected to use the UK standard unit (8 $\mathrm{g}$ alcohol) as the measure of consumption. Participants described units as not "user-friendly", "confusing" and hard to work out to any real degree of accuracy.

"...although we were told what units are what...it can be a bit blurry [especially] if you're drinking cocktails and things like that" (female, opt out, VN550032). "[... it] is quite hard to track. I mean everyone brings a bottle and pours it in [...] it was very hard to judge the amount everyone was drinking" (male, opt in, VN550037).

\section{Intervention delivery and content}

The study was conducted at a time where several educational interventions were on-going (safe drinking limits were advertised on posters around campus). Participants were asked their view on alcohol interventions generally, in relation to student culture and for their views concerning SMS delivered interventions. Existing interventions were ignored:

"I wouldn't say I have [seen intervention material] in [Study Town], [...] there's a little sticker on my mirror that says 'you're looking at the person responsible for your health [...] I'm not sure. There was the odd pamphlet around Fresher's week [...] I probably didn't pay that much attention to it" (male, opt in, VN550029).
Several participants felt "sensory overload" to traditional posters and similar "mass market" campaigns targeting alcohol use, indicating habituation and they further suggested such media were just ignored. Whereas SMS methods were regarded as more appropriate because they were personal.

"[Mass market campaigns are] written to [...] everyone in my age group [they do not reach] out to me personally, so I don't feel the need to respond" (male, opt in, VN550036).

In respect of content, advertising the negative consequences of excessive consumption were seen as aversive for three participants, beneficial for two and the remaining 13 suggested emphasising the positive with the negatives would likely provide the most effective intervention content. The consensus was that content that "grabbed attention" but did not come across as "parenty" was the best overall approach.

"I think [it is better to emphasise the] benefits [...] when [interventions] focus on the negative points it just feels like you're back at home and it's your parents telling you off for drinking too much" (male, opt in, VN550036).

"If you do it both in moderation, the negatives and the positives, it could discourage binge drinking [...] there's been lots of adverts [warning against] heavy drinking and it doesn't seem to be doing much good, students are still drinking as much as ever" (female, opt in, VN550046).

When asked further about potential intervention content, interventions that referred to the financial aspects of alcohol use appealed to the majority of those interviewed, 17 participants, the remaining participants suggested interventions should refer to both health and finance.

"Finance is a big thing for students. I mean it's the biggest conversation I would have thought. Amongst my friends it's always money and how they can save money... so I think tapping into finance would be a really good idea for students" (male, opt out, VN550038).

"I think everyone is aware of their health, health and what to do, but most don't care. Like smoking and drinking and taking drugs...whilst they're young as well, a lot of people have the mentality that 'I'm going to die of something so I might as well have a good time 'til I do"' (female, opt out, VN550040).

The qualitative study revealed that students are happy with SMS delivered messages, as they are perceived as 
secure and private. Obstacles included messages that were sent too early in the morning and that emphasised the negative aspects of excessive alcohol consumption. A strong positive theme for intervention content was the financial implications of excessive alcohol use and the opportunity for messages to help students reduce their expenditure. Financial information was therefore used as the content for an SMS delivered simple intervention that would be delivered late morning (Study II).

\section{Study II: A feasibility randomised control trial using SMS text messaging to provide feedback on alcohol expenditure}

Participants were recruited from a panel of individuals $(\mathrm{N}=493)$ who had completed research on an unrelated project but had agreed to be contacted again if further research opportunities arose. None had participated in Study I. Of these, 87 participants consented and were recruited onto the current study (58 male, 43 students), yielding an average FAST score of $5.54(\mathrm{SD}=2.28) ; 86$ participants responded at least once to the daily SMS messages (see Figure 7). One person actively opted out of the study. The average age of students (mean $=22.00$ years, $\mathrm{SD}=3.66)$ was greater than that of non-students $($ mean $=$ 38.49 years, $\mathrm{SD}=14.26 ; \mathrm{t}=7.05, p<0.001)$.

A mixed two (within factor, average daily units consumed before and after the intervention) by two (student and non-student) by two (experimental group: intervention and control) ANOVA yielded a non-significant main effect of experimental group $(\mathrm{F}(1,70)=1.56, p=0.216$, partial $\eta^{2}=0.02$ ). The means presented in Table 1 indicate that three of the four groups showed a reduction in alcohol consumption from baseline to follow-up: Control Students showed a decrease of 5.5\%; Intervention Non-Students showed a decrease of 4\%; Intervention Students showed a decrease of $19 \%$. However, Control Non-Students showed an increase of $17.1 \%$. Sample size estimates for the change in mean on intervention students and assuming $\sigma=1.7$, power analysis for a one-sample mean test, taking preintervention mean as the null and the post-intervention mean as the alternative (see Table 1 ), and with a 0.8 power $(1-\beta)$ to detect significance at $\alpha=0.05$ suggests a sample size of 140 would be sufficient to raise this to significance. There was little evidence to suggest that the expenditure based intervention generalises as well to non-student populations.

\section{Discussion}

While recruitment successfully attracted a large number of students into Study I, numerous factors appear to have contributed to attrition: the qualitative study indicated that some participants appeared to have signed up to the study without considering what they were signing up to; this was further compounded by technical problems. Irrespective of the reasons for this attrition, no systematic relationship between FAST score and whether or not participants completed the first online survey was observed. This suggests that attrition was not systematically related to hazardous alcohol use. Participation in the repeated online surveys showed a heightened level of attrition; only $33 \%$ of those who completed the first online survey completed the final one, undermining this medium as a means of collecting long-term data when prompted with text messages to do so. Conversely, the number of respondents to the SMS messages remained static over time, once technical issues had been resolved. SMS messages are generally more acceptable, a conclusion also supported in the qualitative research.

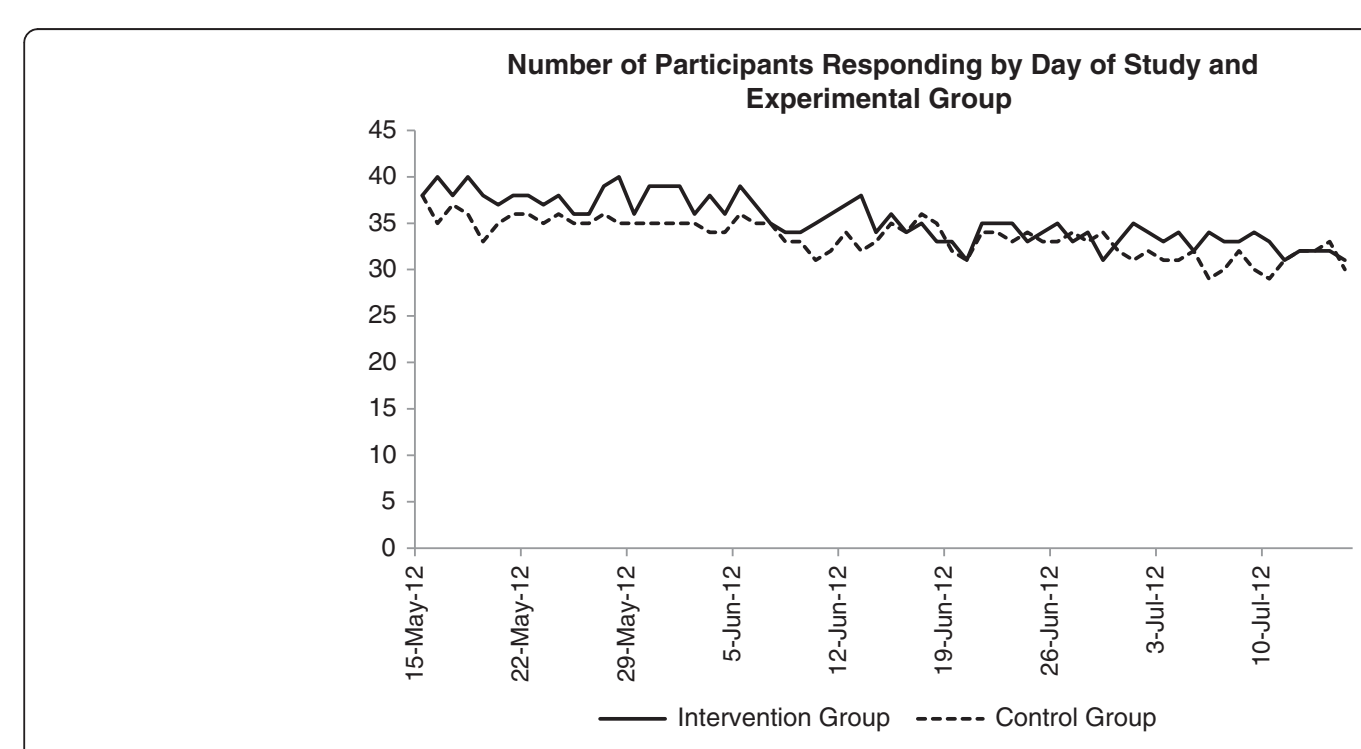

Figure 7 Mean number of participants responding to text messages by day of study and experimental group. 
Table 1 Mean number of units consumed before and after intervention delivery in control and experimental groups

\begin{tabular}{lllrrr}
\hline & Condition & & \multicolumn{3}{c}{ Units } \\
\cline { 3 - 5 } & & & N & Mean & SD \\
\hline Control & Before & Non-student & 22 & 1.29 & 1.06 \\
Control & Before & Student & 16 & 1.63 & 1.65 \\
Control & After & Non-student & 22 & 1.51 & 1.21 \\
Control & After & Student & 14 & 1.54 & 1.34 \\
Intervention & Before & Non-student & 19 & 1.76 & 1.43 \\
Intervention & Before & Student & 21 & 1.89 & 1.67 \\
Intervention & After & Non-student & 18 & 1.69 & 1.81 \\
Intervention & After & Student & 20 & 1.53 & 1.27 \\
\hline
\end{tabular}

FAST scores showed reasonable test re-test reliability and external validity against the AUDIT, suggesting the online delivery of this metric initiated by SMS messages is valid. It is therefore feasible to use the FAST in future trials rather than the more time consuming AUDIT. Those who signed up to the study and went forward and actively responded to SMS messages yielded higher FAST scores compared to those who signed up but did not respond to SMS messages. This may indicate that those who consume alcohol more hazardously are more interested in having their consumption monitored. Furthermore, average units consumed across the study were positively associated with FAST scores, but not the likelihood of drinking, suggesting that the SMS data at least provides a valid index of hazardous alcohol use, consistent with other studies assessing the reliability of self-report data [32].

In further exploration, it was found that the more alcohol consumed the less likely participants were to consume alcohol the following day. The reasons for this association are unclear; it may be due to regret elicited through over-consumption, budgetary constraints or they may have been still feeling the effects of intoxication. Further, there was a positive association between the quantity of alcohol consumed and the time taken to respond to SMS messages the following day. If this delay is also associated with more salient behaviour then alcohol consumption might also be associated with diligence in respect of student activity more generally, in as far that they may also delay their working day.

Alcohol consumption was greatest on Fridays and Saturdays, consistent with previous North American studies $[19,33,34]$, except for one notable exception: Wednesdays were the heaviest drinking weekday, rather than Thursday $[19,20]$. The dissimilarity in weekday drinking suggests a UK university-specific cultural influence. Across UK universities Wednesday afternoons are almost always free from academic commitments in order to facilitate pastoral activities, notably sports. This is traditionally followed by social events during which alcohol is typically consumed [35]. January saw a trough in alcohol consumption and this may be associated with the examinations held during this time. Research from North America has also found drinking moderates during times of greater academic demand [19]. We would therefore recommend that FAST is used to initially triage patients and determine eligibility for the intervention then SMS can be used to ascertain when individuals are most vulnerable to misuse alcohol and in turn direct further intervention.

An exploratory randomised controlled trial was implemented to test the feasibility of a simple intervention relaying estimated expenditure on alcohol for the past month. Having improved recruitment materials so that participants were more aware of the study requirements and worked with SMS providers to insure a faultless delivery of text messages, attrition from the RCT was negligible; suggesting that the identified barriers to study entry had been overcome. The simple intervention developed and deployed in the exploratory RCT yielded effects broadly consistent with expectations but not to significance. The effect appeared to be more salient in student participants for whom the intervention was developed suggesting that the goal-specific intervention would require further development in order for it to generalise.

\section{Conclusions}

Text messaging is an acceptable method of collecting data relating to alcohol consumption. SMS further offers a potentially low-cost vehicle for the rapid delivery of interventions and in a format that is more likely to be noticed compared to posters, leaflets and email communication. The portability of mobile phones also provides an opportunity for real-time data collection. For example, it may be feasible to deliver "in-the-moment" interventions at times when young people are planning to drink alcohol.

The current study assessed a single text message relaying information on alcohol-related expenditure. A next logical stage would be to consider how variations in message content and number might affect change. Feasible content of such interventions may include financial information relating to expenditure on alcohol in addition to healthspecific information for a student population. Further study should consider other forms of novel content in various populations in order to reduce alcohol-related harm. For example, in the student population the intervention may focus on the financial benefits of moderating consumption, perhaps by sending an automated message containing an estimation of how much money they have spent so far during their night out or over longer periods. In other populations, such as those attending Emergency Departments as a result of alcohol-related harm, the content of a text message intervention may contain a warning 
about the potential harm that could result if a person continues to drink.

Our data support the findings of Kuntsche and Robert [18], demonstrating that SMS can be used to collect alcohol consumption data and extends their observations to suggest that this method is an acceptable means of collecting high frequency data over several months. This is particularly valuable for studies with students, as students often do not limit their drinking to weekends. Furthermore, this method may meet the recognised need for methods that are able to characterise drinking style [36], in particular risky single occasion drinking. Two-way SMS text messaging provides an open and available platform well suited to collecting high frequency data from large numbers of individuals simultaneously. Moreover, SMS text messaging may also prove useful for vulnerable young people, for whom SMS text messaging may be the only electronic text based communication available and reinforced through observations that there is a positive association between mobile phone use and detrimental health-related behaviours in 14-16 year olds, including alcohol and tobacco use [37].

Although the purpose of the current study was to develop, test and assess the feasibility of simple interventions delivered using the SMS platform, there were several limitations that a Phase II [25] exploratory trial could address. The first is the nature of the intervention to be delivered. While SMS is popular [21] questions remain on whether information could be regarded as an intervention in its own right, or whether SMS could be used to support face-to-face counselling.

As mobile technology develops, the incorporation of social media including Twitter and Facebook seems likely to become commonplace and therefore underpin the continued growth of short message services including text messaging and instant messaging. Developing surveillance and intervention services that can be adapted for SMS and instant messaging offers a means to identify where and when heavy drinking is taking place. One of the key benefits of using SMS to collect data and deliver interventions is the ability to provide an automated, costeffective service. The finding that SMS is deemed acceptable in this context holds promise for the development of its use in other situations where efficiency of time and resources are paramount, but intervention is greatly needed.

\section{Competing interests}

The authors declare that they have no competing interests.

\section{Authors' contributions}

SCM conceived of the studies in this paper, participated in their design, carried out the statistical analysis of studies 1 and 4, and drafted a large portion of the manuscript. KC participated in the design, acquisition of data, and analysis of Study I, and drafted this portion of the manuscript. SvG and MvdB participated in the design of Study I and critically revised the manuscript. JB conducted the secondary analysis of the alcohol consumption data detailed in Study I, and drafted this portion of the manuscript. EL participated in the design, acquisition of data, and drafting of the studies in this manuscript. All authors read and approved the final manuscript.

\section{Acknowledgements}

This work was supported by the European Foundation for Alcohol Research, European Research Advisory Board (EA0904). We would like to thank James Murphy, Janette Baird and Brian Suffoletto for their patience and for critically appraising earlier drafts of the manuscript.

\section{Author details}

${ }^{1}$ Violence \& Society Research Group, School of Dentistry, Cardiff University, CF14 4XY Cardiff, Wales. ${ }^{2}$ Department of Applied Psychology, Cardiff Metropolitan University, Llandaff Campus, Western Avenue, CF5 2YB Cardiff, Wales. ${ }^{3}$ School of Psychology, Cardiff University, 70 Park Place, CF10 3AT Cardiff, Wales. ${ }^{4}$ MRC Centre for Neuropsychiatric Genetics \& Genomics, Cardiff University School of Medicine, Neuadd Meirionnydd, Heath Park, CF14 4YS Cardiff, Wales. ${ }^{5}$ Cardiff University School of Medicine, Heath Park, CF14 4YU Cardiff, Wales.

Received: 24 January 2013 Accepted: 21 October 2013

Published: 25 October 2013

\section{References}

1. World Data Bank: Mobile cellular subscriptions (per 100 people); 2011. Archived by WebCite at http://www.webcitation.org/62EclQ7q7.

2. Nielsen: In U.S., SMS Text Messaging Tops Mobile Phone Calling; 2012. Archived by WebCite at http://www.webcitation.org/62EcoS3VX.

3. Fjeldsoe BS, Marshall AL, Miller YD: Behavior change interventions delivered by mobile telephone short-message service. Am J Prev Med 2009, 36(2):165-173.

4. Cunningham JA, Kypri K, McCambridge J: The use of emerging technologies in alcohol treatment. Alcohol Res Health 2011, 33(4):320-326.

5. Depp CA, Mausbach B, Granholm E, Cardenas V, Ben-Zeev D, Patterson TL, Lebowitz BD, Jeste DV: Mobile interventions for severe mental illness: design and preliminary data from three approaches. J Nerv Ment Dis 2010, 198(10):715-721.

6. Koivusilta LK, Lintonen TP, Rimpelä AJ: Orientations in adolescent use of information and communication technology: A digital divide by sociodemographic background, educational career, and health. Scand J Public Health 2007, 35(1):95-103.

7. Merlo L, Ahmedani BK, Barondess DA, Bohnert KM, Gold MS: Alcohol consumption associated with collegiate American football pre-game festivities. Drug \& Alcohol Dependence 2011, 116(1-3):242-245.

8. Bischof G, Grothues JM, Reinhardt S, Meyer C, John U, Rumpf H-J: Evaluation of a telephone-based stepped care intervention for alcohol-related disorders: A randomized controlled trial. Drug Alcohol Depend 2008, 93(3):244-251.

9. Ackerman MJ, Filart R, Burgess LP, Lee I, Poropatich RK: Developing nextgeneration telehealth tools and technologies: patients, systems, and data perspectives. Telemedicine and e-Health 2010, 16(1):93-95.

10. Kypri K, Langley JD, Saunders JB, Cashell-Smith ML, Herbison P: Randomized Controlled Trial of Web-Based Alcohol Screening and Brief Intervention in Primary Care. Arch Intern Med 2008, 168(5):530-536.

11. Bewick BM, Trusler K, Barkham M, Hill AJ, Cahill J, Mulhern B: The effectiveness of web-based interventions designed to decrease alcohol consumption - a systematic review. Preventative Medicine 2008, 47(1):17-26.

12. Cunningham JA, Wild TC, Cordingley J, Van Mierlo T, Humphreys K: A randomized controlled trial of an internet-based intervention for alcohol abusers. Addiction 2009, 104(12):2023-2032.

13. Carey KB, Scott-Sheldon LAJ, Elliott JC, Bolles JR, Carey MP: Computerdelivered interventions to reduce college student drinking: a metaanalysis. Addiction 2009, 104(11):1807-1819.

14. Rooke S, Thorsteinsson E, Karpin A, Copeland J, Allsop D: Computerdelivered interventions for alcohol and tobacco use: a meta-analysis. Addiction 2010, 105(8):1381-1390.

15. Atun R, Sittampalam S: A review of the characteristics and benefits of SMS in delivering healthcare. In The Role of Mobile Phones in Increasing Accessibility and Efficiency in Healthcare Report. Berkshire,UK: Vodafone; 2006.

16. Haberer JE, Kiwanuka J, Nansera D, Wilson IB, Bangsberg DR: Challenges in using mobile phones for collection of antiretroviral therapy adherence data in a resource-limited setting. AIDS Behav 2010, 14(6):1294-1301.

17. Moonen B, Cohen JM: Text messaging to improve adherence to malaria guidelines. Lancet 2011, 378(9793):750-752. 
18. Kuntsche E, Robert B: Short message service (SMS) technology in alcohol research- A feasibility study. Alcohol Alcohol 2009, 44(4):423-428.

19. Tremblay PF, Graham K, Wells S, Harris R, Pulford R, Roberts SE: When do firstyear college students drink most during the academic year? An internetbased study of daily and weekly drinking. J Am Coll Health 2010, 58(5):401-411.

20. Del Boca FK, Darkes J, Greenbaum PE, Goldman MS: Up close and personal: temporal variability in the drinking of individual college students during their first year. Journal of Consulting \& Clinical Psychology 2004, 72(2):155-164.

21. Suffoletto B, Callaway C, Kristan J, Kraemer K, Clark DB: Text-message-based drinking assessments and brief interventions for young adults discharged from the Emergency Department. Alcohol Clin Exp Res 2012, 36(3):552-560.

22. Serre F, Fatseasa M, Debrabanta R, Alexandrea J-M, Auriacombea M, Swendsenc J: Ecological momentary assessment in alcohol, tobacco, cannabis and opiate dependence: A comparison of feasibility and validity. Drug Alcohol Depend 2012, 126(1-2):118-123.

23. Piasecki TM, Alley KJ, Slutske WS, Wood PK, Sher KJ, Shiffman S, Heath AC: Low sensitivity to alcohol: relations with hangover occurrence and susceptibility in an ecological momentary assessment investigation. J Stud Alcohol Drugs 2012, 73(6):925-932.

24. Weitzel JA, Bernhardt JM, Usdan S, Mays D, Glanz K: Using wireless handheld computers and tailored text messaging to reduce negative consequences of drinking alcohol. J Stud Alcohol Drugs 2007, 68(4):534-537.

25. Craig P, Dieppe P, Maclntyre S, Mitchie S, Nazareth I, Petticrew M: Developing and evaluating complex interventions: the new Medical Research Council guidance. Br Med J 2008, 337:979-983.

26. Hodgson R, Alwyn T, John B, Thom B, Smith A: The fast alcohol screening test. Alcohol Alcohol 2002, 37(1):61-66.

27. Babor TF, Higgins-Biddle JC, Saunders JB, Monteiro M: The Alcohol Use Disorders Identification Test: Guidelines for Use in Primary Care. Geneva: World Health Organization: Department of Mental Health and Substance Dependence; 2001.

28. Kaner E, Bland M, Cassidy P, Coulton S, Dale V, Deluca P, Gilvarry E, Godfrey C, Heather N, Myles J, et al: Effectiveness of screening and brief alcohol intervention in primary care (SIPS trial): pragmatic cluster randomised controlled trial. Br Med J 2013, 346:e8501.

29. Barrett B, Byford S, Crawford MJ, Patton R, Drummond C, Henry JA, Touquet $R$ : Cost-effectiveness of screening and referral to an alcohol health worker in alcohol misusing patients attending an accident and emergency department: a decision-making analysis. Drug and Alcohol Depenence 2006, 81:47-54

30. Braun V, Clarke V: Using thematic analysis in psychology. Qual Res Psychol 2006, 3(2):77-101.

31. Bright MA, O'Connor D: Qualitative data analysis: comparison between traditional and computerized text analysis. The Osprey Journal of Ideas and Inquiry 2007:21. Archived by WebCite at http://www.webcitation.org/6KYTuGd5N.

32. Northcote J, Livingston M: Accuracy of self-reported drinking: observational verification of 'last occasion' drink estimates of young adults. Alcohol Alcohol 2011, 46(6):709-713.

33. Beets MW, Flay BR, Vuchinich S, Li K-K, Acock A, Snyder FJ: Longitudinal patterns of binge drinking among first year college students with a history of tobacco use. Drug Alcohol Depend 2009, 103(1-2):1-8.

34. Greenbaum PE, Del Boca FK, Darkes J, Wang C-P, Goldman MS: Variation in the drinking trajectories of freshmen college students. Journal of Consulting \& Clinical Psychology 2005, 73(2):229-238.

35. Martens MP, Dams-O'Connor K, Beck NC: A systematic review of college student-athlete drinking: Prevalence rates, sport-related factors, and interventions. J Subst Abuse Treat 2006, 31(3):305-316.

36. Gmel G, Kuntsche E, Rehm J: Risky single occasion drinking: bingeing is not bingeing. Addiction 2011, 106(6):1037-1045.

37. Leena K, Tomi L, Arja R: Intensity of mobile phone use and health compromising behaviours - how is information and communication technology connected to health-related lifestyle in adolescence? J Adolesc 2005, 28:35-47.

\section{doi:10.1186/1471-2458-13-1011}

Cite this article as: Moore et al: A feasibility study of short message service text messaging as a surveillance tool for alcohol consumption and vehicle for interventions in university students. BMC Public Health 2013 13:1011.

\section{Submit your next manuscript to BioMed Central and take full advantage of:}

- Convenient online submission

- Thorough peer review

- No space constraints or color figure charges

- Immediate publication on acceptance

- Inclusion in PubMed, CAS, Scopus and Google Scholar

- Research which is freely available for redistribution 